\title{
Air-conducted and skull-tap cervical vestibular evoked myogenic potentials in determining nerve division involvement in vestibular schwannoma patients
}

\author{
Magdalena Lachowska ${ }^{A-F}$, Paulina Glinka ${ }^{B, C}$, Kazimierz Niemczyk ${ }^{\mathrm{E}, \mathrm{F}}$ \\ Department of Otolaryngology, Medical University of Warsaw, Poland \\ A - research concept and design; $\mathrm{B}$ - collection and/or assembly of data; $\mathrm{C}$ - data analysis and interpretation; \\ $D$ - writing the article; $E$ - critical revision of the article; $F$ - final approval of the article
}

Address for correspondence

Magdalena Lachowska

E-mail: mlachowska@wum.edu.pl

Funding sources

None declared

Conflict of interest

None declared

Received on March 28, 2016

Reviewed on June 19, 2016

Accepted on September 30, 2016

\begin{abstract}
Background. Air-conducted and skull-tap cervical vestibular evoked myogenic potentials (AC-cVEMP and Tap-cVEMP) have been shown to be very promising tools in clinical practice. They are noninvasive, easy to obtain and - importantly - they require little time and the cost of the instruments is low.
\end{abstract}

Objectives. The aim of this study was to evaluate the usefulness of the combined use of AC- and Tap-CVEMPs as a diagnostic tool for advanced assessment of vestibular schwannoma in determining tumor origin, and to investigate whether the results are helpful for a surgeon as an additional source of information about the tumor before surgery.

Material and methods. AC- and Tap-cVEMPs were acquired (with EMG-based biofeedback) from the sternocleidomastoid muscles (SCM) of 30 vestibular schwannoma patients just before surgery. The results were compared to the surgical information about nerve bundle involvement in the tumor and the size of the tumor obtained from magnetic resonance imaging (MRl).

Results. On the tumor side, abnormal corrected amplitude asymmetry ratios were detected in $73.33 \%$ of the patients, abnormalities in P1-latencies in 70\% of the patients, and both in $90 \%$ of the patients. The cervical vestibular evoked myogenic potential (cVEMP) results indicated the affected nerve division to be the inferior in $23.33 \%$ of the patients, the superior in $20 \%$ of the patients, and both in $46.67 \%$ of the patients. No cVEMP abnormalities were found in 10\% of cases. The combined results of both AC- and Tap-CVEMP were significantly compatible with the surgical information about the tumor origin. The number of abnormalities was significantly correlated with the tumor size.

Conclusions. The information provided by the combined application of AC- and Tap-CVEMPs might be useful for a surgeon in presurgical planning, providing more detailed information about the tumor and the affected nerve division in the internal auditory canal. It is not a diagnostic replacement for MRl in vestibular schwannoma patients; however, in our opinion, AC-and Tap-CVEMPs may serve as additional sources of information about the tumor before the surgery.

Key words: vestibular schwannoma, cerebellopontine angle tumor, sternocleidomastoid muscle, vestibular nerve, unilateral hearing loss

DOI

10.17219/acem/65484

\section{Copyright}

Copyright by Author(s)

This is an article distributed under the terms of the

Creative Commons Attribution Non-Commercial License

(http://creativecommons.org/licenses/by-nc-nd/4.0/) 


\section{Introduction}

Vestibular schwannoma is a benign tumor that grows slowly. It arises from the $8^{\text {th }}$ cranial nerve complex, mainly from the vestibular part, pressing on the acoustic branch, but there are cases arising from the acoustic bundle as well. The $8^{\text {th }}$ cranial nerve divides into individual nerves in the lateral part of the internal auditory canal: the cochlear nerve, the superior vestibular nerve and the inferior vestibular nerve. ${ }^{1-3}$ The cochlear bundle starts more anteriorly, and the vestibular ones more posteriorly. The facial nerve courses anteriorly, like the cochlear nerve, but remains superior to it. Going from the fundus of the internal auditory canal to the cerebellopontine angle, the nerves rotate altogether about $90^{\circ}$, so that the cochlear nerve enters the angle more posteriorly, but remains the most inferior. ${ }^{1}$

Due to the division of the vestibulocochlear nerve into individual nerves, the presence of a tumor can affect the function of these nerves depending on which nerve is compressed. This explains the unilateral tinnitus and sensorineural hearing loss, which are the symptoms usually reported by vestibular schwannoma patients. As mentioned earlier, the tumor enlarges slowly; vestibular dysfunction is compensated at the brainstem level, which is why vertigo is not such a frequent complaint. In addition, facial nerve paresis or palsy may also be observed in some patients with facial nerve compression, as it shares the same trajectory as the cochlear nerve in the auditory canal. As the schwannoma grows, it may press on adjacent nerves outside the internal auditory canal and brainstem structures. These are rare but potentially serious consequences, emphasizing the need for the use of sensitive diagnostic tools in patients suspected of potential vestibular schwannoma. In our department, the general protocol is to follow a diagnostic procedure for vestibular or acoustic schwannoma using the standard tests with the complementary addition of airconducted and skull-tap cervical vestibular evoked myogenic potentials (AC-cVEMP and Tap-cVEMP). ${ }^{4}$

Cervical vestibular evoked myogenic potentials (cVEMPs) are short-latency electromyograms that can be elicited by acoustic, vibration or skull-tap stimuli. They measure the vestibulo-colic reflex recorded from the sternocleidomastoid muscle (SCM). In the literature on sensory organs, the saccule is presented as the one responsible for AC-cVEMPs. ${ }^{5,6}$ Since most of the saccular innervation is carried by the inferior vestibular nerve, its function is essential in the generation of AC-cVEMPs. ${ }^{7-9}$ The utricle is presented as the main sensory origin of TapcVEMPs. ${ }^{10-12}$ Brantberg et al. reported that when the function of the superior vestibular nerve is affected, as in vestibular neuritis, skull-tap cVEMPs are more often abnormal than AC-cVEMPs. ${ }^{11}$ Tap-cVEMPs can still be recorded despite selective section of the inferior vestibular bundle. ${ }^{12}$

The rapid development of VEMP-related research and our own recent experience with AC- and Tap-cVEMPs have led us to use cVEMPs as an integral part of our diagnostic battery in various vestibular problems, particularly in patients with vestibular schwannoma. ${ }^{13}$ VEMPs are noninvasive and easy to obtain; also, they require little time and the cost of the instruments is low, which are important advantages in clinical practice nowadays.

Performing both AC-cVEMPs and Tap-cVEMPs may be helpful in identifying the functional integrity of the inferior and superior vestibular bundles of the $8^{\text {th }}$ nerve. The aim of this study was to evaluate the usefulness of combined AC- and Tap-cVEMPs as a diagnostic tool for advanced assessment of vestibular schwannoma in determining the tumor origin. In this study we did not seek a diagnostic replacement test for MRIs in vestibular schwannoma patients; rather, we investigated whether the information from the combined use of AC-cVEMPs and Tap-cVEMPs might be helpful for a surgeon as an additional source of information about the tumor before surgery.

\section{Material and methods}

\section{The subjects and testing protocol}

This prospective study involved 30 patients, 15 females (50\%) and 15 men (50\%), aged from 20 to 57 years (mean age $40.90 \pm 11.05$ years) with diagnosed vestibular schwannoma and with no other otological or neurological problems. The recordings were obtained with each subject lying comfortably on a bed with the upper body elevated $30^{\circ}$. The subjects were instructed to relax and lift their heads during recordings to provide tonic background SCM activity, without shoulder or abdominal muscle activity if possible. At all times a researcher was present, directing the patient to decrease or increase the head lift to correct the contraction level of the muscle and to stay within the selected root mean square (RMS) EMG activity levels, using continuous pre- and poststimulus biofeedback EMG activity monitoring for guidance as described in our previous study. ${ }^{13}$ All the patients were able to perform the head lift without any issues.

The AC- and Tap-cVEMP recordings were performed and analyzed 1-3 days before the surgical tumor removal. The same very experienced otosurgeon performed all the procedures. He was asked to carefully describe the tumor origin and the nerve bundles that were involved in the tumor, and to provide the researchers with this information after surgery. In order to avoid bias in his descriptions, the surgeon was blind to the VEMP results before the operations; likewise, the VEMPs were analyzed before surgery, so the researchers were blind to the surgical information about the tumor origin.

The study was approved by the Medical University of Warsaw Institutional Ethics Committee Review Board and all the participating subjects signed informed consent forms. 


\section{Stimulus and signal acquisition characteristics}

All the cVEMPs were acquired using a SmartEP, a fully computerized 2-channel evoked potential system (Intelligent Hearing Systems Corp., Miami, USA). Surface electrodes were placed on the skin above the SCM. The inverting $(-)$ electrodes were positioned bilaterally on the sternum and the SCM junction, and the non-inverting $(+)$ ones were placed bilaterally on the midpoint of the SCM between the mastoid and the sternum. The ground electrode was placed on the upper part of the sternum. All electrode impedances were $3 \mathrm{k} \Omega$ or less.

In this study, 2 types of stimuli were used. The acoustic stimuli consisted of a single $500 \mathrm{~Hz}$ frequency exact Blackman window tone burst of $5 \mathrm{~ms}$ duration, presented bilaterally. The acoustic stimuli were delivered via ER3a insert earphones (Etymotic Research Inc., Elk Grove Village, USA) at $100 \mathrm{~dB} \mathrm{nHL}$. For the skull-tapping recordings, we used an automated skull-tapping device (Intelligent Hearing Systems Corp., Miami, USA) that was stabilized on the patient's skull with a headband that ensured a fixed distance and contact pressure. In addition, an EMG standardization method integrated into the acquisition software was used to further minimize the variability of the cVEMP recordings, as described in our previous study. ${ }^{13}$ The skull tapper was placed on the skull at 3 locations: at the midline of the forehead for bilateral symmetrical stimulation and signal acquisition, and behind the left and then the right ears on the mastoid process.

Both the acoustic and skull-tapping evoked recordings were acquired using the head-lifting method so that the SCMs were bilaterally contracted as described above. The acquisition parameters were as follows: a sampling period of $400 \mathrm{~ms}$ with $5 \mathrm{~K}$ amplification of the signal, $6 \mathrm{~dB}$ per octave band pass filter, $30 \mathrm{~Hz}$ high-pass cutoff and $1500 \mathrm{~Hz}$ low-pass cutoff. The AC-cVEMPs were collected by averaging 3 sets of 64 sweeps and the Tap-cVEMPs were acquired by averaging 3 sets of 32 sweeps. Fewer sweeps were used to average the Tap-cVEMPs due to the generally larger amplitude of the responses generated by skull tapping compared to the auditory counterpart. For both types of recordings a stimulation rate of $3.1 / \mathrm{s}$ was used.

\section{VEMP data analysis}

The recorded electrophysiological responses were then normalized according to the prestimulus (base) RMS EMG calculations. ${ }^{14-17}$ The resulting VEMP waveforms were analyzed for a response presence for each type of stimulation (acoustic and tapping) in the time domain. The first distinctive positive peak was identified as P1 (or p13), followed by a distinctive negative trough $\mathrm{N} 1$ (or $\mathrm{n} 23$ ). The latencies and amplitudes were measured. Normalized values were used to assess the corrected asymmetry ratios (corrAR) between the left and right side measurements. ${ }^{17}$ Responses from the ear with vestibular schwannoma that were not detected were assigned an amplitude of 0 , which means the corrected amplitude ratio in those cases was $100 \%$. A corrAR $>34 \%$ was considered abnormal. Latencies greater than the mean +2SD of $13.31 \mathrm{~ms}$ (AC-cVEMP) and $10.77 \mathrm{~ms}$ (Tap-cVEMP) were considered abnormal (based upon data collected from 22 healthy ears in subjects without any otological or neurological problems as controls).

\section{Results}

Vestibular schwannoma was diagnosed on 1 side in all the patients, confirmed by MRI. It was found in $12(40 \%)$ patients on the right side and in 18 (60\%) patients on the left side. In maximum diameter, the tumor size ranged from 4 to $37 \mathrm{~mm}$, with the average of $13.80 \mathrm{~mm}( \pm 8.05 \mathrm{~mm})$ and a median of $13.00 \mathrm{~mm}$.

In all healthy ears (those without the tumor), cVEMPs were preserved. In each patient, the healthy side was used as a reference. On the tumor side, in AC- and Tap-cVEMP responses, abnormalities of corrected amplitude asymmetry ratios (corrAR $\geq 35 \%$ ) were detected in 22 out of $30 \mathrm{pa}$ tients (73.33\%), abnormalities in P1 latencies in 21 out of 30 patients (70\%), and either corrAR and/or P1 latency in 27 out of 30 patients $(90 \%)^{18}$

Table 1 shows AC- and Tap-cVEMP results for the vestibular schwannoma side and the healthy ear for all stimulation types. It includes the mean and median values for P1 corrected amplitudes, corrected amplitude asymmetry ratios and P1 latencies. The differences between the tumor side and the healthy side proved to be significant.

Figure 1 shows an example of a set of recordings from 1 vestibular schwannoma patient (tumor size $10 \times 14 \times 8 \mathrm{~mm}$ ). The AC- and Tap-cVEMP averaged waveforms are shown in separate panels. In this patient, the schwannoma was diagnosed on the left side. Visual inspection of the presented waveforms, P1 latency measurements and amplitude calculations revealed the tumor to be located on the superior bundle of the left vestibular nerve. This AC- and Tap-cVEMP result was then confirmed by the surgeon describing the tumor located on that nerve bundle.

Like in the previous example, Fig. 2 shows waveforms recorded from another vestibular schwannoma patient, but this time the tumor was located on the right side and was larger $(21 \times 24 \times 32 \mathrm{~mm})$, involving both vestibular nerve divisions. This tumor location established by the AC- and Tap-cVEMP results was then confirmed by the surgeon.

The combined AC- and Tap-cVEMPs results indicated the affected nerve bundle to be the inferior one in $23.33 \%$ of the patients, the superior one in $20 \%$ of the patients, and both bundles in $46.67 \%$ of the patients. No cVEMP abnormalities were found in $10 \%$ of the patients (Table 2). The surgical information revealed the following tumor locations: inferior vestibular nerve in $40 \%$ of the patients, 
Table 1. Means ( \pm SD) and medians for peak-to-peak amplitudes, corrected amplitude asymmetry ratios (corrAR) of AC- and Tap-cVEMPs in the tumor sites and the healthy ears of vestibular schwannoma patients $(n=30)$

\begin{tabular}{|c|c|c|c|c|c|c|c|c|}
\hline & & & Mean & $\pm \mathrm{SD}$ & Median & Mean & $\pm \mathrm{SD}$ & Median \\
\hline & & & \multicolumn{3}{|c|}{ both ears stimulated at the same time } & \multicolumn{3}{|c|}{$\begin{array}{l}\text { One ear stimulated } \\
\text { (ipsilateral to the recording site) }\end{array}$} \\
\hline \multirow{6}{*}{$\begin{array}{l}\text { AC- } \\
\text {-CVEMP }\end{array}$} & \multirow{2}{*}{$\begin{array}{l}\text { corrected } \\
\text { amplitudes }\end{array}$} & tumor site & 6.93 & 7.24 & 5.27 & 10.32 & 10.79 & 7.06 \\
\hline & & corrAR (\%) & 53.64 & 35.90 & 41.58 & 49.87 & 38.70 & 31.35 \\
\hline & \multirow{4}{*}{ latencies } & healthy site & 13.57 & 0.70 & 13.60 & 13.70 & 0.73 & 13.80 \\
\hline & & tumor site & 13.95 & 1.50 & 13.70 & 13.94 & 0.85 & 13.80 \\
\hline & & latency asymmetry (ms) & 0.88 & 1.25 & 0.40 & 0.79 & 0.69 & 0.60 \\
\hline & & significance ( $p$-value) & \multicolumn{3}{|c|}{0.0854} & \multicolumn{3}{|c|}{$0.0332^{*}$} \\
\hline & & & \multicolumn{3}{|c|}{ forehead tapping } & \multicolumn{3}{|c|}{$\begin{array}{l}\text { One mastoid tapping } \\
\text { (ipsilateral to the recording site) }\end{array}$} \\
\hline \multirow{8}{*}{$\begin{array}{l}\text { Tap- } \\
\text {-cVEMP }\end{array}$} & \multirow{4}{*}{$\begin{array}{l}\text { corrected } \\
\text { amplitudes }\end{array}$} & healthy site & 19.42 & 8.44 & 18.50 & 16.48 & 10.05 & 12.59 \\
\hline & & tumor site & 14.73 & 9.97 & 14.11 & 12.20 & 10.25 & 10.83 \\
\hline & & corrAR (\%) & 30.47 & 24.63 & 23.63 & 27.02 & 29.31 & 17.04 \\
\hline & & significance (p-value) & \multicolumn{3}{|c|}{$0.0196^{*}$} & \multicolumn{3}{|c|}{$0.0300^{*}$} \\
\hline & \multirow{4}{*}{ latencies } & healthy site & 12.41 & 2.09 & 11.90 & 13.29 & 2.81 & 12.80 \\
\hline & & tumor site & 14.23 & 3.20 & 13.20 & 14.58 & 3.46 & 14.40 \\
\hline & & latency asymmetry (ms) & 1.90 & 1.93 & 1.20 & 1.96 & 2.27 & 1.40 \\
\hline & & significance (p-value) & \multicolumn{3}{|c|}{$0.0309^{*}$} & \multicolumn{3}{|c|}{$0.0031^{*}$} \\
\hline
\end{tabular}

* Significant differences $(\mathrm{p}<0.05)$ between the tumor site and healthy site (Wilcoxon signed-rank test).

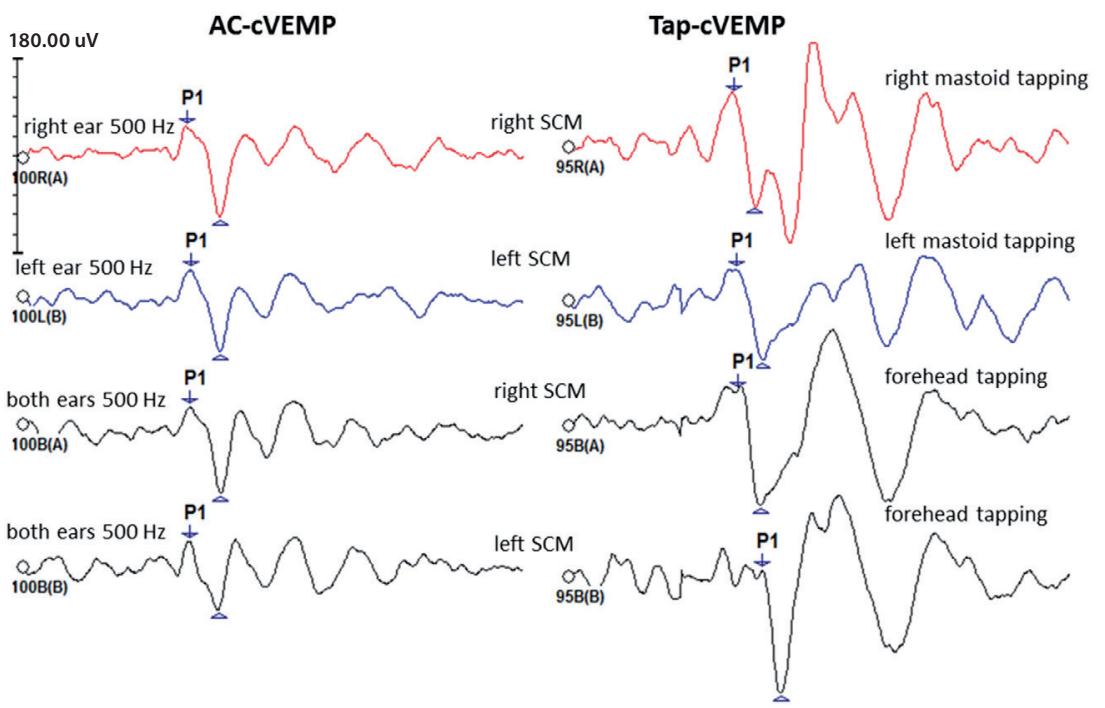

Fig. 1. An example of a set of recordings obtained from 1 vestibular schwannoma patient (tumor size $10 \times 14 \times 8 \mathrm{~mm}$, located on the left side, superior bundle of the vestibular nerve)

AC-cVEMP and Tap-cVEMP averaged waveforms are shown in separate panels. The $1^{\text {st }}$ row in each panel shows responses from the right sternocleidomastoid muscle (SCM); the $2^{\text {nd }}$ shows responses from the left SCM to ipsilateral stimulation (AC stimulus delivered to the ear via inserted earphone and tap stimulus delivered to the mastoid using a skull tapper); the bottom 2 rows show waveforms recorded from the right and left SCM ( $3^{\text {rd }}$ and $4^{\text {th }}$ rows from the top, respectively) to bilateral acoustic stimulation (AC-CVEMP) and to forehead tapping (Tap-cVEMP); in each recording the $\mathrm{P} 1$ wave is marked.

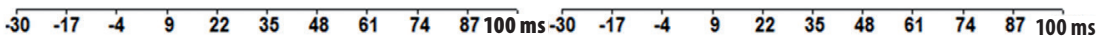

Table 2. Compatibility between the surgical information and AC- and Tap-cVEMP results

\begin{tabular}{|l|c|c|c|}
\multicolumn{2}{|c|}{ AC- and Tap-cVEMP result } & \multicolumn{2}{c}{$\begin{array}{c}\text { Surgical information } \\
\text { (surgical and VEMP) }\end{array}$} \\
\hline \multicolumn{1}{|c|}{ affected nerve } & number of patients & affected nerve & number of patients \\
\hline inferior & 7 & inferior & 12 \\
\hline superior & 6 & superior & 3 \\
\hline both & 14 & both & 12 \\
\hline normal result & 3 & cochlear & 3 \\
\hline
\end{tabular}

The $p$-value represents the level of significance. 


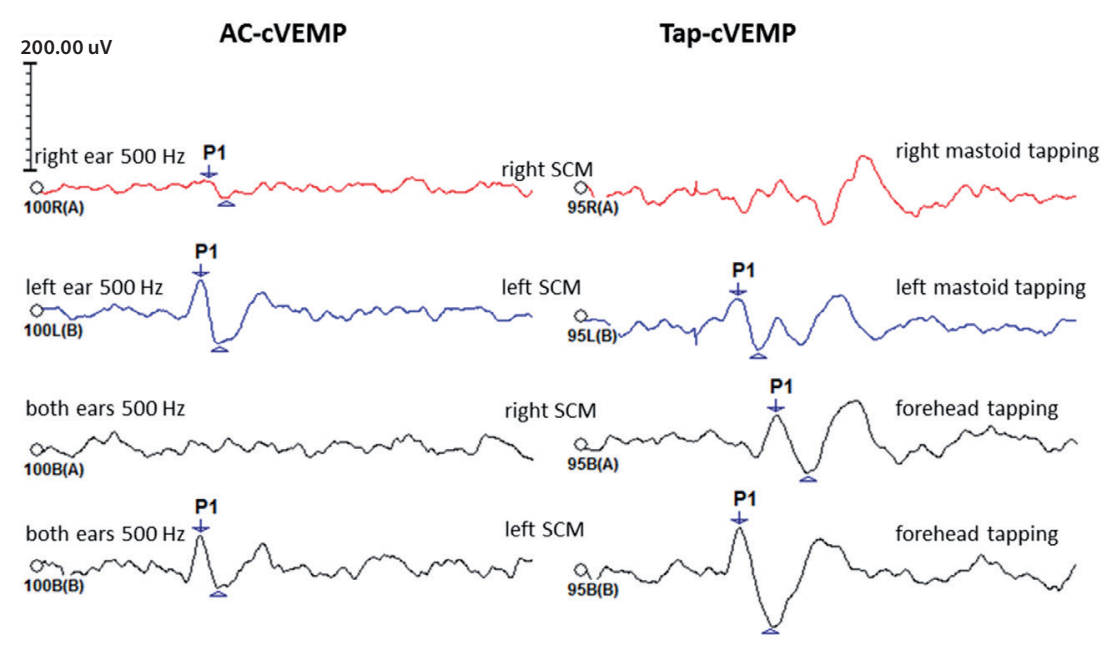

Fig. 2. An example of a set of recordings obtained from 1 vestibular schwannoma patient (tumor size $21 \times 24 \times 32 \mathrm{~mm}$, located on the right side, inferior and superior bundles affected)

AC-CVEMP and Tap-cVEMP averaged waveforms are shown in separate panels. The $1^{\text {st }}$ row in each panel shows responses from the right sternocleidomastoid muscle (SCM); the $2^{\text {nd }}$ shows responses from the left SCM to ipsilateral stimulation (AC stimulus delivered to the ear via inserted earphone and tap stimulus delivered to the mastoid using skull tapper); the bottom 2 rows show waveforms recorded from the right and left SCM ( $3^{\text {rd }}$ and $4^{\text {th }}$ rows from the top, respectively) to bilateral acoustic stimulation (AC-cVEMP) and to forehead tapping (Tap-cVEMP); in each recording the P1 wave is marked.

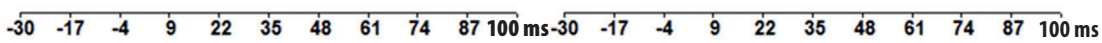

superior in $10 \%$ of the patients, both bundles in $40 \%$ of the patients, and solely cochlear nerve involvement in $10 \%$ of the patients. The combined AC-and Tap-cVEMP results were significantly compatible with the surgical information about the tumor origin $(\mathrm{p}<0.0002)$.

Further useful information was obtained by counting the number of combined AC- and Tap-cVEMP abnormalities on the tumor side for each patient and comparing them to the tumor size. As mentioned before, abnormal cVEMP results were found in 27 out of 30 patients (90\%). Table 3 shows the prevalence of the number of detected abnormalities (corrAR and latency) in AC-and Tap-cVEMPs. In addition, in 27 out of 30 patients (90\%) auditory brainstem response $(\mathrm{ABR})$ results showed retrocochlear origins. Combined AC-cVEMP, Tap-cVEMP and ABR results showed abnormalities in 28 out of 30 patients (93.33\%);

Table 3. Descriptive statistics along with correlations between the tumor size and the number of AC- and Tap-cVEMP detected abnormalities in corrAR and latency measurements

\begin{tabular}{|c|c|c|}
\hline \multicolumn{2}{|c|}{$\begin{array}{c}\text { AC and Tap cVEMP } \\
\text { corrAR }(\%) \text { and latency abnormalities }\end{array}$} & \multirow{2}{*}{$\begin{array}{l}\text { Correlation with } \\
\text { the tumor size }\end{array}$} \\
\hline $\begin{array}{l}\text { number of detected } \\
\text { abnormalities }\end{array}$ & $\begin{array}{l}\text { number } \\
\text { of patients }\end{array}$ & \\
\hline 0 & 3 & \multirow{9}{*}{$p=0.0080$} \\
\hline 1 & 7 & \\
\hline 2 & 6 & \\
\hline 3 & 2 & \\
\hline 4 & 1 & \\
\hline 5 & 4 & \\
\hline 6 & 2 & \\
\hline 7 & 3 & \\
\hline 8 & 2 & \\
\hline
\end{tabular}

corrAR - corrected amplitude asymmetry ratio between the tumor site and the healthy site; $p$-value represents the level of significance. however, the ABR results were not the focus of the present study. The number of combined AC- and Tap-cVEMP abnormalities, as well as the number of combined cVEMP and ABR abnormalities, were significantly correlated with the tumor size $(\mathrm{p}=0.0080$ and $\mathrm{p}=0.0000$, respectively).

\section{Discussion}

In this study, we present a combined approach using both AC- and Tap-cVEMPs to determine nerve division involvement in vestibular schwannoma as an additional source of helpful information for surgeons prior to operations. AC-cVEMPs are generated by saccular activity, and thus reflect mainly the activity of the inferior vestibular nerve. ${ }^{8,12,19}$ On the other hand, it has been suggested that Tap-cVEMPs generate a more complex stimulation paradigm. They have been described as consisting of 2 different mechanisms: the $1^{\text {st }}$ resulting in ipsilateral inhibitory activity on the SCM, and the $2^{\text {nd }}$ producing a bilateral response with opposite polarities for the two SCM muscles. ${ }^{20}$ The utricle has been described as the origin of the unilateral and bilateral components through different mechanisms. ${ }^{20-22}$ This has been supported by other studies as well. ${ }^{12}$ Brantberg and Mathiesen proved that Tap-cVEMPs were preserved despite sectioned inferior vestibular nerves, suggesting the involvement of superior vestibular nerve activity in Tap-cVEMP responses. ${ }^{12}$ Recording both the AC- and Tap-cVEMPs therefore further increases the potential for identifying the affected nerve bundles. Where no Tap-cVEMPs are present but AC-cVEMPs are, that indicates a nonfunctional superior division of the $8^{\text {th }}$ nerve; and in contrast, where no AC-cVEMPs are present but Tap-cVEMPs are, that points to a nonfunctional inferior bundle with a functional superior bundle. The results of our study further support this, showing significant compatibility between AC- and Tap-cVEMP information 
about the affected nerve bundle and surgical information about the tumor origin.

MRI scans provide information on the presence of a tumor, its location in the internal auditory canal and its size. This information is essential for the diagnosis and for a surgeon; however, it does not say which nerve divisions are involved in the pathological process. In our study, the combined information from $\mathrm{AC}$ - and Tap-cVEMP results was significantly correlated with the surgical findings about the tumor origin. The cVEMP results were found to be normal in 3 patients, but in 1 of those patients, ABRs showed retrocochlear disorders (in the remaining 2, the ABRs were normal). This means that in our study, the electrophysiological findings of cVEMPs alone and combined cVEMPs and ABRs were helpful in identifying the tumor origin in $90 \%$ and $93.33 \%$ of the patients, respectively. As mentioned before, in both cases, this information was significantly correlated with the surgical findings. This shows that AC- and Tap-cVEMPs assessment is essential in vestibular schwannoma, and can provide the surgeon with helpful additional information about the probable involvement of nerve bundles in the tumor. This information, combined with ABR results and MRIs, can be helpful in presurgical planning and patient counseling. The electrophysiological tests (cVEMPs and ABRs) show how much residual vestibular and cochlear function is present prior to surgery, providing the surgeon with information that is very useful in decisions about additional intraoperative monitoring of hearing. In our department, intraoperative monitoring of hearing is routinely used in every vestibular schwannoma surgery performed via the middle fossa approach and in many other ear operations; however, hearing is not routinely monitored intraoperatively in most other oto- or neurosurgical facilities. Electrophysiological results might be useful when counseling patients and informing them of realistic possible outcomes of surgery, such as the chances of preserving hearing, the risk of hearing loss during surgery, or if a patient is likely to develop vertigo after the tumor removal and therefore require vestibular rehabilitation. However, postoperative management was not analyzed in this study.

The results presented in this study show a good correlation between the tumor size and the number of abnormalities detected in AC-and Tap-cVEMPs, considering corrAR and the latencies of the responses. Those results further support the findings of Lin et al. and Taylor et al., who also found significant correlations between their tests results and tumor size. ${ }^{23,24}$ However, they used ocular vestibular evoked myogenic potentials (oVEMPs) to investigate the superior vestibular nerve division instead of Tap-cVEMPs. In our department, cVEMPs are used due to their longer history and more extensive research, but recently we have also been investigating the usefulness of oVEMPs. Although recent research on oVEMPs has been encouraging in terms of clinical practice, the technique of this vestibular testing method still needs to be refined to be used as a routine clinical test. Cervical VEMPs are quite easy to obtain and can be efficiently used with quantification methods for ongoing muscle activity monitoring. With constant EMG monitoring, the amplitudes can easily be normalized according to the prestimulus (base) RMS calculations, as in this study. In the near future, we plan to include oVEMPs in our routine diagnostic tests in patients with vestibular problems, ensuring that the adaptation of muscle activity quantification methods we use is properly carried out. ${ }^{14-17}$

This article describes our VEMP findings in 30 vestibular schwannoma patients from a still growing data collection which we plan to present in the near future. We believe that further investigations in vestibular schwannoma patients are needed to confirm the clinical utility of AC-cVEMPs and Tap-cVEMPs as presurgical tests providing additional information to a surgeon about the nerve bundles involved in the pathological process. Currently, we are collecting ocular VEMP data (using both air-conducted and boneconducted stimuli) from a growing patient population. In the near future, when sufficient patient population data has been collected, we plan to analyze different combinations of AC-cVEMP, Tap-cVEMP and oVEMP to evaluate the clinical usefulness of different testing protocols. Our attempt to establish the most clinically useful VEMP testing protocol will be presented in a subsequent paper.

In conclusion, the information provided by the combined application of AC- and Tap-cVEMPs can be useful to surgeons in presurgical planning, providing detailed information about the tumor and the affected nerve division in the internal auditory canal. MRI scans provide information on the presence of a tumor, its location in the internal auditory canal and its size, which is essential for the diagnosis and for the operation, but it does not provide information about the nerve divisions involved in the pathological process. In our opinion, AC- and Tap-cVEMPs may serve as additional sources of information about the tumor before surgery.

\section{References}

1. Silverstein $\mathrm{H}$. Cochlear and vestibular gross and histologic anatomy (as seen from postauricular approach). Otolaryngol Head Neck Surg. 1984;92(2):207-211.

2. Schefter RP, Harner SG. Histologic study of the vestibulocochlear nerve. Ann Otol Rhinol Laryngol. 1986;95(2 Pt 1):146-150.

3. Rubinstein D, Sandberg EJ, Cajade-Law AG. Anatomy of the facial and vestibulocochlear nerves in the internal auditory canal. AJNR Am J Neuroradiol. 1996;17(6):1099-1105.

4. Sterkers O, Martin C, Arnold W. Vestibular schwannoma. In: Anniko M, Bernal-Sprekelsen M, Bonkowsky V, Bradley P, lurato S, eds. Otorhinolaryngology, Head and Neck Surgery - European Manual of Medicine. Leipzig: Springer Verlag; 2010:92-97.

5. Young ED, Fernandez C, Goldberg JM. Responses of squirrel monkey vestibular neurons to audio-frequency sound and head vibration. Acta Otolaryngol. 1977;84(5-6);352-360.

6. Murofushi T, Curthoys IS. Physiological and anatomical study of clicksensitive primary vestibular afferents in the guinea pig. Acta Otolaryngol. 1997;117(1):66-72.

7. Townsend GL, Cody DT. The averaged inion response evoked by acoustic stimulation: Its relation to the saccule. Ann Otol Rhinol Laryngol. 1971;80(1):121-131. 
8. Murofushi T, Halmagyi GM, Yavor RA, Colebatch JG. Absent vestibular evoked myogenic potentials in vestibular neurolabyrinthitis. An indicator of inferior vestibular nerve involvement? Arch Otolaryngol Head Neck Surg. 1996;122(8):845-848.

9. Murofushi T, Matsuzaki M, Mizuno M. Vestibular evoked myogenic potentials in patients with acoustic neuromas. Arch Otolaryngol Head Neck Surg. 1998;124(5):509-512.

10. Brantberg K, Tribukait A. Vestibular evoked myogenic potentials in response to laterally directed skull taps. J Vestib Res. 2002;12(1):35-45.

11. Brantberg K, Tribukait A, Fransson PA. Vestibular evoked myogenic potentials in response to skull taps for patients with vestibular neuritis. J Vestib Res. 2003;13(2-3):121-130.

12. Brantberg K, Mathiesen T. Preservation of tap vestibular evoked myogenic potentials despite resection of the inferior vestibular nerve. J Vestib Res. 2004;14(4):347-351.

13. Yavuz E, Lachowska M, Pierchała K, Morawski K, Niemczyk K, Delgado RE. Clinical use of skull tap vestibular evoked myogenic potentials for the diagnoses of the cerebellopontine angle tumor patients. BioMed Res Int. 2014;2014:135457. doi:10.1155/2014/135457

14. Colebatch JG. Properties of rectified averaging of an evoked-type signal: Theory and application to the vestibular-evoked myogenic potential. Exp Brain Res. 2009;199(2):167-176.

15. McCaslin DL, Jacobson GP, Hatton K, Fowler AP, DeLong AP. The effects of amplitude normalization and EMG targets on cVEMP interaural amplitude asymmetry. Ear Hear. 2013;34(4):482-490.

16. van Tilburg MJ, Herrmann BS, Guinan JJ Jr, Rauch SD. Normalization reduces intersubject variability in cervical vestibular evoked myogenic potentials. Otol Neurotol. 2014;35(8):222-227.
17. Papathanasiou ES, Murofushi T, Akin FW, Colebatch JG. International guidelines for the clinical application of cervical vestibular evoked myogenic potentials: An expert consensus report. Clin Neurophysiol. 2014;125(4):658-666.

18. Welgampola MS, Colebatch JG. Vestibulocollic reflexes: Normal values and the effect of age. Clin Neurophysiol. 2001;112(11):19711979.

19. Tsutsumi T, Tsunoda A, Noguchi Y, Komatsuzaki A. Prediction of the nerves of origin of vestibular schwannomas with vestibular evoked myogenic potentials. Am J Otol. 2000;21(5):712-715.

20. Brantberg K, Westin M, Löfqvist L, Verrecchia L, Tribukait A. Vestibular evoked myogenic potentials in response to lateral skull taps are dependent on two different mechanisms. Clin Neurophysiol. 2009; 120(5):974-979.

21. Kushiro K, Zakir M, Ogawa Y, Sato H, Uchino Y. Saccular and utricular inputs to sternocleidomastoid motoneurons of decerebrate cats. Exp Brain Res. 1999;126(3):410-416.

22. Todd NP, Rosengren SM, Colebatch JG. A source analysis of shortlatency vestibular evoked potentials produced by air-and bone-conducted sound. Clin Neurophysiol. 2008;119(8):1881-1894.

23. Lin $\mathrm{KL}$, Chen CM, Wang SJ, Young YH. Correlating vestibular schwannoma size with vestibular-evoked myogenic potential results. Ear Hear. 2014;35(5):571-576.

24. Taylor RL, Kong J, Flanagan S, et al. Prevalence of vestibular dysfunction in patients with vestibular schwannoma using video headimpulses and vestibular-evoked potentials. J Neurol. 2015;262(5): 1228-1237. 